PROCEEDINGS OF THE

AMERICAN MATHEMATICAL SOCIETY

Volume 86, Number 1, September 1982

\title{
ON ZERO SETS FOR THE BALL ALGEBRA ${ }^{1}$
}

\author{
H. ALEXANDER
}

\begin{abstract}
Rudin has constructed a function in the ball algebra in $\mathbf{C}^{2}$ whose zero set has infinite volume and has asked if such functions exist in $\mathbf{C}^{n}$. Using some recent work of Ryll and Wojtaszczyk on homogeneous polynomials we shall extend Rudin's proof to $\mathbf{C}^{n}$ for arbitrary $n$.
\end{abstract}

1. Introduction. Let $A\left(B_{n}\right)$ denote the ball algebra, that is, the space of functions holomorphic on the open unit ball $B_{n}$ in $\mathbf{C}^{n}$ and continuous on its closure. For $n=2$, Rudin [1, p. 139] has given an example of a function $f$ in the ball algebra whose zero set in $B_{2}$ has infinite volume (= area, in this case). His method involves a construction with monomials in one complex variable and Rudin has asked [1, pp. 139, 414] if such a result holds for $n>2$, even for an $H^{\infty}$ function.

Recently Ryll and Wojtaszczyk [2] have proved the existence of homogeneous polynomials in $n$ complex variables whose $L^{2}$ and $L^{\infty}$ norms on $\partial B_{n}$ are comparable, independent of their degrees. Jointly in [2] and also in [3] they have shown that certain results in one complex variable can be extended to several complex variables by replacing monomials in the one-variable constructions with their special homogeneous polynomials in the several variable case. The object of this note is to apply this approach to Rudin's question. We shall adapt Rudin's proof for $n=2$ to prove:

THEOREM. For all $n$, there exists an $f \in A\left(B_{n}\right)$ whose zero set in $B_{n}$ has infinite $2 n-2$ dimensional volume.

Professor Rudin has informed me that E. Amar has obtained a like result by other methods.

2. A construction in $B_{n}$. According to Ryll and Wojtaszczyk [2] there exist homogeneous polynomials $f_{k}$ in $\mathbf{C}^{n}$ of degree $k$ for $k=1,2,3, \ldots$, such that $\left\|f_{k}\right\|_{\infty}=1$ and $\left\|f_{k}\right\|_{2} \geq q$ where the norms are the $L^{\infty}(\sigma)$ and $L^{2}(\sigma)$ norms for $\sigma$ the normalized invariant measure on $\partial B_{n}$ and $q>0$ depends only on $n$, not on $k$.

Choose $a_{j}, j=1,2,3, \ldots$ such that

(a) $q / 4<a_{j}<q / 3$ and

(b) $a_{j}$ is a regular value for $f_{j}$; i.e. $d f_{j} \neq 0$ on $\left\{f_{j}=a_{j}\right\}$.

Now define, for $z \in B_{n}$,

$$
P(z)=\prod_{j=1}^{\infty}\left(1-\frac{f_{2^{j}}}{a_{2^{j}}}\right) .
$$

Then $P(0)=1$ and $P$ has multiplicity one on its zero set by (b). From $\left\|f_{j}\right\|_{\infty}=1$ it follows that $\left|f_{2^{j}}(z)\right| \leq\|z\|^{2^{j}}$ for all $z$, where $\|z\|$ is the Euclidean norm of $z$.

Received by the editors November 5, 1981.

1980 Mathematics Subject Classification. Primary 32A40, 32A22; Secondary 32A99.

${ }^{1}$ Supported in part by a grant from the National Science Foundation.

(c) 1982 American Mathematical Society 0002-9939/81/0000-0389/802.25 
This and $1 / a_{2^{j}}<4 / q$ imply

$$
|P(z)| \leq \prod_{j=1}^{\infty}\left(1+\left(\frac{4}{q}\right)\|z\|^{2^{j}}\right) \leq 1+\sum_{t=1}^{\infty}\left(\frac{4}{q}\right)^{c(t)}\|z\|^{t}
$$

where $c(t)$ is the number of ones in the binary representation of $t$ (this argument follows Rudin [1, p. 138]). From $2^{c(t)} \leq t+1$ we get

$$
|P(z)| \leq 1+\sum_{t=1}^{\infty}(t+1)^{N}\|z\|^{t} \leq \frac{\text { constant }}{(1-\|z\|)^{N+1}}
$$

where $N$ is an integer satisfying $N>\log (4 / q) / \log 2$. Now define $f$ on $B_{n+1}$ by

$$
f\left(z, z_{n+1}\right)=z_{n+1}^{2 N+3} P(z)
$$

with $z=\left(z_{1}, z_{2}, \ldots, z_{n}\right)$. Since $\left|z_{n+1}\right| \leq\left(1-\|z\|^{2}\right)^{1 / 2}$ in $B_{n+1}$, the last estimate for $P(z)$ implies that $f$ is in the ball algebra $A\left(B_{n+1}\right)$. We shall prove the theorem by showing that the zero set of $f$ in $B_{n+1}$ has infinite $2 n$ dimensional volume.

3. The growth of the zero set of $P(z)$. For $r<1$ and $F$ holomorphic on $B_{n}$ we set

and put, for $\alpha \in \partial B_{n}$,

$$
N(F, r)=\int \log |F(r \alpha)| d \sigma(\alpha)
$$

$$
N(F, r, \alpha)=\frac{1}{2 \pi} \int_{-\pi}^{\pi} \log \left|F\left(r e^{i \theta} \alpha\right)\right| d \theta .
$$

Then $N(F, r)=\int N(F, r, \alpha) d \sigma(\alpha)$. We have

$$
N(P, r)=\sum_{j=1}^{\infty} N\left(1-\frac{f_{2^{j}}}{a_{2^{j}}}, r\right)=\sum_{j=1}^{\infty} \int N\left(1-\frac{f_{2^{j}}}{a_{2^{j}}}, r, \alpha\right) d \sigma(\alpha)
$$

The following fact is standard.

LEMMA. $1 /(2 \pi) \int \log \left|1-\varsigma \lambda^{n}\right| d \theta=\log ^{+}|\zeta|$ where $\lambda=e^{i \theta}, n=1,2,3, \ldots$

This gives

$$
\begin{aligned}
N\left(1-\frac{f_{2^{j}}}{a_{2^{j}}}, r, \alpha\right) & =\int_{-\pi}^{\pi} \log \left|1-\frac{r^{2^{j}} f_{2^{j}}(\alpha)}{a_{2^{j}}} \cdot e^{i 2^{j} \theta}\right| \frac{d \theta}{2 \pi} \\
& =\log ^{+}\left(\frac{r^{2^{j}}\left|f_{2^{j}}(\alpha)\right|}{a_{2^{j}}}\right)
\end{aligned}
$$

Hence

$$
\begin{aligned}
N(P, r) & =\sum_{j=1}^{\infty} \int \log +\left(\frac{r^{2^{j}}\left|f_{2^{j}}(\alpha)\right|}{a_{2^{j}}}\right) d \sigma(\alpha) \\
& \geq \sum_{j=1}^{\infty} \int_{\left\{\alpha:\left|f_{2^{j}}(\alpha)\right| \geq 2 a_{2^{j}} / r^{2^{j}}\right\}} \log ^{+}\left(\frac{r^{2^{j}}\left|f_{2^{j}}(\alpha)\right|}{a_{2^{j}}}\right) d \sigma(\alpha) \\
& \geq \log 2 \sum_{j=1}^{\infty} \sigma\left\{\alpha:\left|f_{2^{j}}(\alpha)\right| \geq \frac{2 a_{2^{j}}}{\left.r^{2^{j}}\right\} .}\right.
\end{aligned}
$$


LEMMA. For $0<b<q$ and for all $k$,

$$
\sigma\left\{\alpha:\left|f_{k}(\alpha)\right| \geq b\right\} \geq \frac{q^{2}-b^{2}}{1-b^{2}} \equiv \delta(b)>0 .
$$

Proof. Let $E_{k}=\left\{\alpha:\left|f_{k}(\alpha)\right| \geq b\right\}$. Then

$$
q^{2} \leq \int\left|f_{k}\right|^{2} d \sigma=\int_{E_{k}}+\int_{\partial B_{n} \backslash E_{k}} \leq \sigma\left(E_{k}\right)+b^{2}\left(1-\sigma\left(E_{k}\right)\right) .
$$

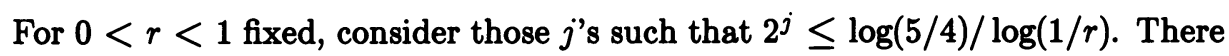
are at least

$$
J(r) \equiv \log \left(\frac{\log 5 / 4}{\log 1 / r}\right) / \log 2-1
$$

such $j$ 's. Then $(1 / r)^{2^{j}} \leq 5 / 4$ and so $2 a_{2^{j}} / r^{2^{j}} \leq 2 \cdot 5 / 4 \cdot q / 3=5 q / 6$. The previous lemma implies

$$
\sigma\left\{\left|f_{2^{j}}\right| \geq \frac{2 a_{2^{j}}}{r^{2^{j}}}\right\} \geq \sigma\left\{\left|f_{2^{j}}\right| \geq \frac{5}{6} q\right\} \geq \delta\left(\frac{5 q}{6}\right)>0 .
$$

We get $N(P, r) \geq J(r) \delta(5 q / 6)$. Because $J(r) \sim \log (1 /(1-r)) / \log 2$ as $r \rightarrow 1$, it follows that $N(P, r) \rightarrow \infty$ as $r \rightarrow 1$; hence

$$
\sup _{0<r<1} \int \log |P(r \alpha)| d \sigma(\alpha)=\infty \text {. }
$$

4. The zero set of $f$. Let $V=Z(P) \equiv\left\{z \in B_{n}: P(z)=0\right\}$. Let $\pi_{n+1}$ be the projection from $B_{n+1}$ to $B_{n}: \pi_{n+1}\left(z, z_{n+1}\right)=z$ for $z \in B_{n}$. Let $\tilde{V}=$ $\pi_{n+1}^{-1}(V)=\left\{\left(z, z_{n+1}\right) \in B_{n+1}: z \in V\right\}$. Then recalling $f\left(z, z_{n+1}\right)=z_{n+1}^{2 N+3} P(z)$ we have $Z(f)=\tilde{V} \cup W$ where $W=\left\{(z, 0): z \in B_{n}\right\}$.

LEMMA. Let $A$ be an analytic subvariety of $B_{n}$ of pure dimension $d$ and let $\pi_{n+1}^{-1}(A)$ be the corresponding $d+1$ dimensional subvariety of $B_{n+1}$. Then

$$
(2 d+2)-\operatorname{vol}\left(\pi_{n+1}^{-1}(A)\right)=\pi \int_{A}\left(1-\|z\|^{2}\right) d v(z)
$$

where $v$ is the $2 d$ volume measure on $A$.

ProOF. For $d+1$ dimensional subvarieties of $\mathbf{C}^{n}$ the volume form is given by

$$
\frac{1}{(d+1) !}\left(\frac{i}{2} \partial \bar{\partial}\|z\|^{2}+\frac{i}{2} \partial \bar{\partial}\left|z_{n+1}\right|^{2}\right)^{d+1} \text {. }
$$

On varieties of the type $\pi_{n+1}^{-1}(A)$ this reduces to

$$
\begin{array}{r}
\frac{1}{(d+1) !}\left[(d+1)\left(\frac{i}{2} \partial \bar{\partial}\|z\|^{2}\right)^{d} \wedge \frac{i}{2} \partial \bar{\partial}\left|z_{n+1}\right|^{2}\right] \\
=\left(\frac{1}{d !}\left(\frac{i}{2} \partial \bar{\partial}\|z\|^{2}\right)^{d}\right) \wedge \frac{i}{2} \partial \bar{\partial}\left|z_{n+1}\right|^{2}
\end{array}
$$

This last expression is the product measure on $\pi_{n+1}^{-1}(A)$ and, since the fibre of $\pi_{n+1}^{-1}(A)$ over a point $z \in A$ is a disc of radius $\left(1-\|z\|^{2}\right)^{1 / 2}$, the result follows by Fubini's theorem. 
It is known (cf. $[1, \S 17.3 .6]$ ) that there are several equivalent ways to formulate the Blaschke condition in the ball $B_{n}$. In particular, the conditions

$$
\sup _{0<r<1} \int_{\partial B_{n}} \log |P(r \alpha)| d \sigma(\alpha)<\infty
$$

and

$$
\int_{Z(P)}\left(1-\|z\|^{2}\right) d v<\infty
$$

are equivalent. We have shown that (a) does not hold. Hence the integral in (b) does not converge. By the previous lemma we conclude that the $2 n$-volume of $\tilde{V}=$ $\pi_{n+1}^{-1}(V)$ is infinite and consequently that the zero set of $f$ in $B_{n+1}$ has infinite volume. This completes the proof of our theorem.

REMARK. Implicit in this argument is the fact that the previous lemma implies that, for a subvariety $V$ of $B_{n}$ with $\tilde{V}=\pi_{n+1}^{-1}(V), \tilde{V}$ in $B_{n+1}$ has finite volume if and only if $V$ satisfies the Blaschke condition in $B_{n}$.

\section{REFERENCES}

1. Walter Rudin, Function theory in the unit ball of $\mathbf{C}^{n}$, Springer-Verlag, New York, 1980.

2. J. Ryll and P. Wojtaszczyk, On homogeneous polynomials on a complex ball, preprint.

3. P. Wojtaszczyk, On functions in the ball algebra, preprint.

Department of Mathematics, University of Illinois at Chicago Circle, BoX 4348, CHICAGO, ILLINOIS 60680 